\title{
TURISMO Y CAMBIO SOCIOCULTURAL EN LA COMUNIDAD LOCAL: UNA APROXIMACIÓN ETNOGRÁFICA EN LA RIVIERA NAYARIT, MÉXICO
}

\author{
Rafael Hernández Espinosa, Dr. en Antropología \\ Universidad Autónoma del Estado de México \\ Martha Marivel Mendoza Ontiveros, Dra. en Ciencias Antropológicas \\ Universidad Autónoma del Estado de México \\ Leonardo Héctor Rioja Peregrina, Dr. en Ciencias Políticas \\ Universidad de Quintana Roo, México
}

\section{Resumen}

El artículo presenta resultados derivados de un proyecto de investigación sobre impactos socioculturales de Centros Integralmente Planeados (CIPs), en las comunidades receptoras en México. En particular, se analizan las significaciones sobre el turismo en general y sobre un complejo turístico reciente asistido por el Fondo Nacional de Fomento al Turismo (FONATUR). Mediante un diseño metodológico cualitativo que incluyó entrevistas y observación etnográfica en la comunidad de Higuera Blanca, en el estado de Nayarit, se observó que la influencia del turismo es percibida de forma heterogénea y relativa por los habitantes dependiendo de las modalidades del mismo. Los hallazgos sugieren que, al no tener aún una participación directa ni tampoco intensa en la actividad turística de la región en general, ni en el complejo colindante en particular, los habitantes de Higuera Blanca construyen significados particulares sobre sus efectos; asociados con beneficios económicos relativos pero también con experiencias de segregación y exclusión social.

Palabras clave: Turismo, CIP, impactos sociales, exclusión social 


\title{
TOURISM AND SOCIOCULTURAL CHANGE IN LOCAL COMMUNITY: AN ETHNOGRAPHIC APPROACH IN THE RIVIERA NAYARIT, MEXICO
}

\author{
Rafael Hernández Espinosa, Dr. en Antropología \\ Universidad Autónoma del Estado de México \\ Martha Marivel Mendoza Ontiveros, Dra. en Ciencias Antropológicas \\ Universidad Autónoma del Estado de México \\ Leonardo Héctor Rioja Peregrina, Dr. en Ciencias Políticas \\ Universidad de Quintana Roo, México
}

\begin{abstract}
This paper presents results derived from a research project on sociocultural impacts of Integrally Planned Centers (CIPs) in receiving communities in Mexico. Particularly, we analyze meanings about tourism in general and about a recent tourist complex assisted by the National Fund for Tourism Promotion (FONATUR) in particular. Through a qualitative methodological design, which included interviews and ethnographic observation in the community of Higuera Blanca, state of Nayarit, it was observed that the inhabitants, depending on the modalities of tourism, perceive the influence of it in a heterogeneous and relative way. Findings suggest that, because they do not have yet a direct or intense participation in tourist activity of this region in general, or in neighboring complex in particular, inhabitants of Higuera Blanca construct particular meanings about their effects; associated with relative economic benefits but also with experiences of segregation and social exclusion.
\end{abstract}

Keywords: tourism, CIP, social impacts, social exclusion 


\section{Introducción}

En este texto se presentan resultados derivados de un proyecto de investigación sobre los impactos socioculturales del turismo en Centros Integralmente Planeados en México, específicamente del caso de estudio del CIP Riviera Nayarit, México. El texto se concentra en las significaciones que la comunidad de Higuera Blanca tiene sobre el turismo y sobre sus efectos en la misma. Higuera Blanca es la comunidad más próxima al proyecto turístico integral Litibú, que forma parte del CIP, por lo que ha sido testigo de su origen y evolución, además del desarrollo de la actividad turística general de la región. Los datos se generaron mediante observación etnográfica, entrevistas y conversaciones informales con habitantes de la comunidad en una primera aproximación etnográfica realizada en el mes de abril de 2016 y en una segunda estancia en abril de 2017. Higuera Blanca pertenece al municipio de Bahía de Banderas, el cual conforma el territorio de mayor desarrollo turístico en la costa sur del estado de Nayarit. En este sentido, dicha comunidad se halla inmersa en un entorno de progresivo desarrollo turístico en diversas dimensiones que condiciona su dinámica económica, social y cultural.

No obstante, la influencia del turismo en la comunidad es relativamente indirecta y se da a partir de cuatro modalidades: el turismo tradicional masificado en los años 60 cuya principal influencia es el Puerto de Vallarta, los turistas de segunda residencia (en su mayoría extranjeros) en un asentamiento aledaño a su comunidad, el complejo Litibú, perteneciente al CIP Nayarit, desarrollado recientemente por el Fondo Nacional de Fomento al Turismo (FONATUR) y los vacacionistas locales que periódicamente vistan la playa Litibú. Los hallazgos sugieren que, al no mantener una participación directa ni tampoco intensa en la actividad del CIP, en particular, ni con la actividad turística de la región, en general, como otras localidades aledañas, los habitantes de Higuera Blanca generan percepciones y significaciones particulares sobre sus efectos en la comunidad. Estas percepciones y significaciones muestran un conjunto de aspectos relevantes, no sólo en torno al turismo, sino de procesos sociales, políticos, económicos, históricos y culturales. Destacan en particular los contrastes entre los relativos beneficios derivados del desarrollo turístico y las experiencias de desigualdad, despojo y exclusión social. El análisis de este aspecto paradójico se aborda mediante una perspectiva hermenéutica que permitió, más que identificar y enlistar los impactos del turismo, comprender los procesos que derivan en la construcción de determinadas significaciones y su vínculo con pautas de acción social. 


\section{Hacia un estudio interpretativo de los procesos socioculturales en comunidades inmersas en contextos turísticos}

Debido a que, a lo largo del tiempo, el turismo ha demostrado no ser la industria sin chimeneas que se pregonaba, surgen demandas de la población y de la academia para que se consideren los impactos a lo largo de la planificación, la gestión y en el proceso de evaluación de sus efectos con el propósito de que los beneficios sean optimizados y los problemas minimizados. Una adecuada política turística de planificación debe buscar reforzar los efectos positivos y mitigar los negativos. Asimismo, estudios han evidenciado que la participación de las comunidades en la planificación, con el objetivo de que los residentes entiendan al turismo y tomen parte en las decisiones, ha llevado a que se reciban mayores beneficios (Kavallinis y Pizam 1994, McIntyre, Hetherington y Inskeep, 1993 citado en Brunt y Courtney 1999). Por lo anterior, la atención académica se ha centrado desde la década de los 70 en los impactos del turismo, en general, y en la comprensión de las percepciones y las actitudes de las poblaciones residentes sobre él. En 1982 Mathieson y Wall señalaron que aunque muchos estudios hacían referencia a la presencia de impactos sociales, tanto positivos como negativos, la mayoría arrojaba poca luz sobre su naturaleza, por tal motivo recomendaron que la investigación debía ser dirigida más explícitamente a la determinación de las percepciones y actitudes por lo que han aparecido numerosos estudios centrados en ellas (Choi y Murray, 2010; Vargas, Porras y Plaza, 2011; Yu, Chancellor y Cole, 2011; Woosnam, 2012; ZamaniFarahani y Musa, 2012; Chen y Raab, 2012; Hunter, 2013; Parra-Camacho y Bastías, 2013; Kim, Uysal y Sirgy, 2013; Lee, 2013; Nunkoo, Smith y Ramkissoon, 2013; Hunt y Stronza, 2014; Mendoza Ontiveros y González Sosa, 2014; Cardona y Serra Cantallops, 2014 Monterrubio, Sosa y Josiam, 2014; Rodrigues Marins, Feder Mayer y Fratucci, 2015; Arias-Hidalgo y Méndez-Estrada, 2015; Bassi y Cardona, 2015; Milito, Juniro y Alexandre, 2015; Sánchez-Fernández y Cardona, 2016; Ramón Cardona y Álvarez Bassi, 2016; Berrezueta, Grunauer, Carrillo, Contreras, y García, 2017, Jiménez, Méndez y Rodríguez, 2017).

La necesidad de explicar y predecir las percepciones y las actitudes de la población local hacia el turismo llevó, en la década de los 90, a adoptar la teoría del intercambio social con el objetivo de medir el apoyo turístico en diferentes circunstancias, momentos y productos. El punto de partida para el intercambio social viene de la necesidad de ser recíprocos en relación a los beneficios obtenidos, con el objeto de continuar recibiéndolos (Northcote y Macbeth 2005). De esta forma, la teoría plantea que una sociedad local que encuentra que el intercambio le favorece en su bienestar, mostrará interés en apoyar el turismo y tendrá respuestas positivas en relación a los turistas. Por el contrario, los residentes que ven el intercambio como problemático se opondrán al desarrollo del turismo. No obstante, las críticas principales van en el sentido de que las percepciones 
de los residentes pueden estar influidas por varios factores sociales y psicológicos que pueden limitar o incluso distorsionar las percepciones de las personas sobre el costo y los beneficios obtenidos. Por ejemplo, la criminalidad puede ser percibida como un impacto negativo del turismo, pero puede en realidad estar causada por factores tales como un cambio sociodemográfico, incremento en los niveles de ingresos o algunas prácticas de la policía.

Sin embargo, a pesar del volumen significativo y el alcance creciente de la investigación, sigue siendo incierto hasta qué punto se ha mejorado la comprensión de las percepciones de los residentes sobre el turismo. Al destacar los temas y tendencias clave de la literatura, se identifican una serie de limitaciones en la investigación, incluyendo una estrecha base de estudios de casos, una dependencia de los métodos cuantitativos, un enfoque en las percepciones en lugar de las respuestas y la exclusión del turista en la mayoría de la investigación. Es común que en el estudio de las percepciones se utilicen encuestas a gran escala en forma de cuestionario que combinan una variedad de formatos de preguntas y se distribuyen por correo o por medio de una "entrevista" estructurada cara a cara. Estos métodos cuantitativos describen qué impactos son percibidos por los residentes, pero no necesariamente dicen por qué tales percepciones (Northcote y Macbeth 2005).

En consonancia con la tradición positivista, la teoría del intercambio social está estrechamente vinculada a la teoría de la elección racional (Fishbein y Ajzen, 1975). Ambas conciben al individuo como un homo economicus, hombre racional que cuenta con información completa, persigue su propio interés, quiere maximizar los beneficios y reducir los costos. Es decir, un sujeto será racional cuando es capaz de ponderar la relación entre costos y beneficios que espera recibir de la acción que emprende, lo cual significa que no realizará ninguna acción si cree que no recibe algún beneficio. No obstante, como sostuvo Max Weber (1922a), la plena conciencia de las acciones es un caso límite y en la inmensa mayoría de las situaciones, los individuos son parcialmente conscientes de lo que hacen. La acción real sucede la mayor parte de los casos con oscura semi-consciencia o plena inconsciencia de su "sentido nombrado". El agente más bien "siente" de un modo indeterminado que "sabe" o tiene clara idea; actúa, en la mayor parte de los casos, por instinto o costumbre. Sólo ocasionalmente algunos individuos tienen conciencia del sentido (sea racional o irracional) de la acción.

Hacer un estudio sobre los impactos o efectos sociales de una actividad, igualmente social, implica en efecto, hacer un estudio de las percepciones. Es decir, resulta necesario involucrar la dimensión subjetiva de los miembros de esa sociedad en torno a esa actividad, pues la dinámica social se enlaza invariablemente con una dimensión simbólica. No obstante, es importante ir más allá de una simple identificación de "las percepciones", pues una perspectiva similar asume que estas suelen ser reflejos, 
más o menos filtrados, de las realidades dadas. Ante ello Weber sostendría que lo esencialmente social de las acciones humanas está en una dimensión simbólica (1922b).

Las teorías antropológicas y sociológicas que convergieron posteriormente en el interés por este aspecto contribuyeron ampliamente a profundizar en el tema desde perspectivas simbólicas y semióticas. Desde ahí, una larga tradición en la teoría social se desarrollado sobre la premisa de que las acciones y las prácticas sociales tienen detrás de sí sentidos específicos que se enlazan con la construcción de significados en torno a las situaciones, los otros sujetos y los objetos de la realidad en general (Blumer, 1969; Schutz, 1932; Garfinkel, 1967; Berger y Luckman, 1968). En cuanto a la dimensión cultural, Clifford Geertz ha brindado, acorde con esta perspectiva, una de las definiciones de cultura más influyentes en tanto se concibe como "sistema de concepciones heredadas y expresadas en formas simbólicas por medios con los cuales los hombres comunican, perpetúan y desarrollan su conocimiento y sus actitudes frente a la vida" (1973, pág. 88).

Con base en lo anterior, este estudio se ha propuesto analizar, más allá de la identificación de las percepciones de los efectos de la actividad turística, la construcción de significados sobre esta actividad y su relación con las acciones y prácticas sociales que dan forma a determinados procesos sociales y culturales en una comunidad particular.

\section{El contexto: la Riviera Nayarit y la Bahía de Banderas}

La Riviera Nayarit, es un constructo discursivo que remite a un espacio geográfico con carácter turístico decretado oficialmente en el 2007 por el gobierno del estado de Nayarit. Este espacio, con una extensión de 180.18 kilómetros de litoral, entre el margen derecho de la desembocadura del Río Ameca hasta el Puerto de San Blas, comprende zonas de desarrollo turístico de los municipios costeros de Bahía de Banderas, Compostela y San Blas (Márquez, Ocampo y Ramos, 2010). Sin embargo, la evolución de la actividad turística en esta región tiene antecedentes en el despunte de Puerto Vallarta varias décadas atrás.

Fue a inicios de la década de 1960 cuando Puerto Vallarta comenzó a tomar importancia en cuanto al desarrollo turístico y el sector servicios. A partir de ahí, esa reorientación en la vocación económica comenzó a impactar en las áreas de influencia, implicando la paulatina sustitución del sector agrícola (Fernández Agraz, 2014). Esta transformación, había iniciado en la década anterior con los efectos de la implantación del programa federal denominado "La marcha al mar", el cual promovía el desarrollo costero y la explotación moderna de los recursos marítimos del país. Ello, por extensión, involucró el desarrollo del sector servicios y del turismo en Puerto Vallarta. El Pacífico mexicano de occidente se transfiguraría así en el foco de desarrollo turístico, posicionándose como 
un conjunto de destinos de sol y playa.

Paulatinamente, la actividad turística fue extendiéndose sobre la Bahía de Banderas y el sur de Nayarit. Algunas comunidades costeras, como Sayulita, comenzaron a recibir visitantes extranjeros atraídos por sus características pintorescas y bellezas naturales, y otras más fueron generándose a partir de la inversión en hoteles y servicios turísticos. Sin embargo, es importante subrayar que en diciembre de 1970 el gobierno federal expropió una gran cantidad de terrenos a lo largo de la franja costera del sur de Nayarit, por considerarlos de utilidad pública para el desarrollo habitacional y turístico (Secretaría del Patrimonio Nacional, 1970). Ello sugiere que, desde entonces, esta región se había concebido como ideal para el futuro desarrollo turístico ${ }^{1}$

El conjunto de predios expropiados pasó entonces a formar parte del Fideicomiso Bahía de Banderas (FIBBA) que finalmente sería administrado por el gobierno del estado de Nayarit hasta 1989 para darle un impulso decidido a su desarrollo turístico. Para esta época, la región de la bahía había entrado en auge económico y en 1989 el gobierno del estado decretó la creación de un nuevo municipio, el número veinte, en lo que fuera antes la parte sur del municipio de Compostela y que llevaría por nombre "Bahía de Banderas" (Gobierno del Estado de Nayarit, 1989). La creación del municipio proporcionó, de tal suerte, una mejor gestión administrativa del territorio (Fernández Agraz, 2014) y a su vez permitió un mayor impulso del desarrollo turístico, facilitándose la inversión internacional (Salazar Peralta, 2013).

En Nayarit, el turismo se había desarrollado a partir de la década de los 70, concentrándose principalmente en el mercado nacional. Ello se había logrado construyendo infraestructura urbana, favoreciendo la expropiación y legalización de tierras ejidales, construyendo inmuebles para uso hotelero y aportando financiamiento para empresarios y para capacitación de fuerza de trabajo (Fernández Agraz, 2014). De esta forma, poblaciones como Rincón de Guayabitos, Lo de Marcos, San Francisco, Sayulita y Bucerías se fueron constituyendo como lugares de atracción turística.

1 Cabe mencionar que, a mediados de ese año los entonces presidentes de México y de Estados Unidos, Gustavo Díaz Ordaz y Richard Nixon, habían celebrado una reunión de jefes de estado en Puerto Vallarta (Fernández Agraz, 2014). 
Figura 1. Distribución demográfica en la región de la Bahía de Banderas

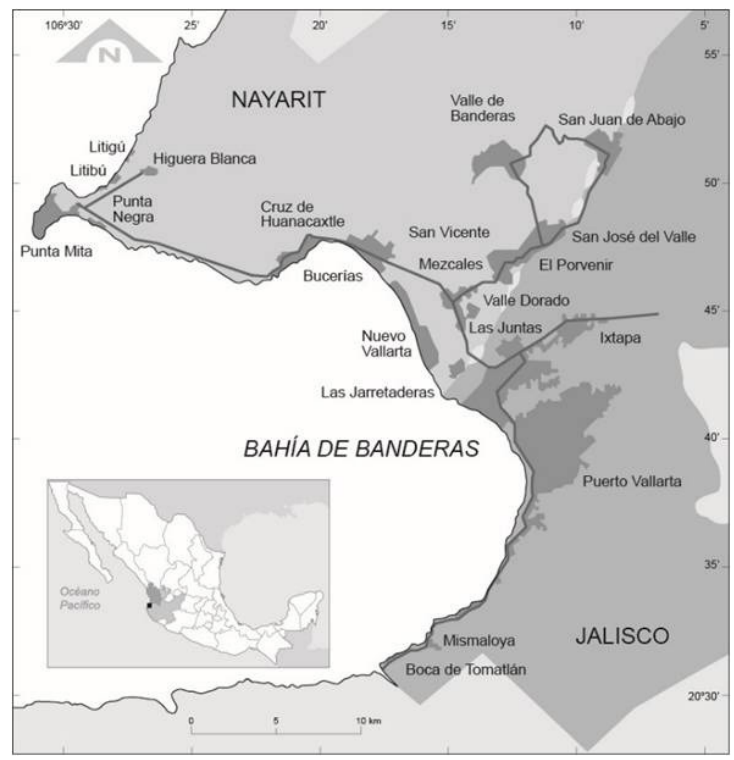

Fuente: Baños Francia (2014)

Ya en la década de los ochenta se comenzaron a desarrollar otros espacios turísticos importantes, como Nuevo Vallarta en los límites con Jalisco, que incluyó un campo de golf así como hoteles y fraccionamientos exclusivos (Fernández Agraz, 2014) y que darían a la zona de la bahía una imagen de desarrollo turístico mayor. Es importante notar que aunque el mercado mayoritario para entonces era nacional, principalmente de la región occidente, la zona se fue también trasformando en un destino atractivo para los turistas norteamericanos y en especial para la población jubilada. Como menciona Ana María Salazar (2013), con el impulso del desarrollo turístico se buscó también la satisfacción de demanda del turismo residencial de los baby boomers norteamericanos, es decir, el segmento representado por personas en etapa jubilatoria que cuenta con capacidad económica y que busca un sitio para vivir una vida saludable. De esa forma se comenzó a establecer un fenómeno de atracción poblacional extranjera temporal, el turismo de segundas residencias. En Bahía de Banderas, este tipo de turismo se ha desarrollado a partir de las inversiones públicas y sobre todo privadas, reflejadas en la construcción de hoteles de cinco estrellas y comunidades resort que están orientadas a un segmento de alto poder adquisitivo, pues sus cuotas son excéntricamente elevadas y están destinadas a un ambiente de exclusividad (Salazar Peralta, 2013). 
Dos de estos resorts exclusivos, se encuentran en lo que sería una especie de microrregión, situada entre las comunidades de Emiliano Zapata, Corral del Risco e Higuera Blanca, en el extremo poniente de la Bahía de Banderas. Los complejos turísticos de Punta Mita y Litibú fueron desarrollados, respectivamente, por las dos grandes empresas turísticas y de condominios de la región: DINE, de carácter privado, y el Fondo Nacional de Fomento al Turismo (FONATUR), del sector público federal (Fonseca, 2009). En la primera mitad de la década de 1990 se consolidó el proyecto Punta Mita, un gran complejo turístico que incluye un campo de golf así como hoteles y condominios exclusivos. Vale la pena señalar que para la construcción de este proyecto se desalojó y reubicó una población que habría quedado dentro de los límites del polígono. Por otro lado, a mediados de la década del dos mil, el FONATUR inició la construcción de la primera fase del Centro Integralmente Planeado (CIP) Nayarit, y que corresponde al polígono Litibú, en un predio anexo a la comunidad de Higuera Blanca. El proyecto original del CIP comprende tres polígonos o Proyectos Turísticos Integrales (PTI) en diferentes puntos de la Riviera Nayarit: el polígono "Litibú", en el municipio de Bahía de Banderas, y en el municipio de Compostela los polígonos "Costa Capomo" y "La Peñita de Jaltemba”. De ellos, Litibú es el primero en desarrollarse puesto que corresponde a la primera fase (FONATUR, 2006). El polígono que comprende este proyecto tiene 167 hectáreas de superficie destinadas para usos hoteleros, residenciales y comerciales, así como para un club y campo de golf de 18 hoyos (Moro Ingeniería S.C., 2002). Ambos desarrollos son ejemplos de complejos desarrollados bajo un modelo de turismo de enclave, es decir de acceso exclusivo y diseñado para un mercado nacional e internacional de alto gasto.

Como se puede observar, con base en lo descrito hasta ahora, en la región hay actualmente una variedad de destinos de sol y playa, así como de servicios turísticos. Sin embargo, es innegable que la tendencia actual presenta una marcada tendencia hacia el desarrollo de un turismo de enclave que se ofrece a un público exclusivo, preferentemente extranjero, y de alto gasto. Ello ha venido configurando cambios interesantes en este territorio, tanto en términos físico-ambientales, como económicos, sociales y culturales.

La región, por otro lado, ha transitado hacia un importante proceso de terciarización, transformando la forma de vida de los habitantes originarios, e integrado a nuevos actores que continúan arribando permanentemente por motivos laborales. Las empresas industriales que predominan en Bahía de Banderas están vinculadas con la actividad turística y representan el $87.6 \%$ de las unidades económicas (Ayuntamiento de Bahía de Banderas, 2014). Otro de los cambios importantes que ha habido en la región en los últimos años es el acelerado crecimiento demográfico. Bahía de Banderas, fue el municipio nayarita con mayor aumento de habitantes en la primer década del presente siglo (Gómez, 2010), mostrando una elevación en la tasa de crecimiento poblacional de 4.2 a 7.7 entre el 2000 y el 2010 (Fernández Agraz, 2014). En 2010 el municipio contaba 
con un total de 124 mil 205 habitantes, que representaron el 8.73\% de la población correspondiente al estado de Nayarit. Diversos estudiosos coinciden en que este fenómeno está directamente relacionado con la inmigración generada por la atracción de mano de obra asociada al turismo (Fonseca, 2009; Gómez, 2010 y Salazar Peralta, 2013, Fernández Agraz, 2014). En general, el sur de Nayarit atrae tanto migrantes nacionales (jornaleros agrícolas, trabajadores de la construcción, mujeres para el trabajo doméstico; y personal para servicios turísticos y profesionistas) como extranjeros, que buscan condiciones climáticas y ambientales deseables para pasar el invierno (Gómez, 2010).

En síntesis, la zona sur de la Riviera Nayarit se halla en una dinámica de transformación económica y sociocultural determinada fundamentalmente por la actividad turística. A nivel de las localidades estas transformaciones se expresan de diversas formas, pues cada una tiene orígenes y vínculos con este proceso distintos. No obstante, La comunidad de Higuera Blanca es un caso interesante por varias razones, entre ellas porque a uno de sus costados se construyó el proyecto Litibú, del CIP Nayarit.

Según datos del cronista del municipio de Bahía de Banderas, Eduardo Gómez Encarnación (2014), esta comunidad fue uno de los varios ranchos que pertenecieron a la Hacienda de Jaltemba, la cual se dedicaba a la ganadería y la explotación del "coquito de aceite", dada la gran cantidad de palmares que crecían de manera natural en la región. Aunque no se ofrecen datos de su fundación, Gómez Encarnación (2015) señala que a fines de la década de los 1930 Higuera Blanca contaba con sólo tres casas. Para 1950, el séptimo Censo General de Población reportó que Higuera Blanca contaba ya con 88 habitantes (Secretaría de economía, 1952). Por esta época la población de la región continuaba dedicándose a la producción del aceite de coco, que vendían a las fábricas de aceite que se ubicaban en Mazatlán, Tepic, Guadalajara y Compostela (Gómez Encarnación, 2014).

Por otra parte, aunque el reparto agrario en la región había iniciado a finales de los 30, en el poblado de Sayulita (Departamento agrario, 1940), no fue sino hasta 1951 que se dotó de tierras ejidales a Higuera Blanca (Departamento agrario, 1953), sin embargo las condiciones de vida y de acceso no mejoraron pronto. Algunos habitantes mayores señalan que llegaron a la comunidad hace cerca de 60 años, provenientes de otros lugares de Nayarit, atraídos por la disponibilidad de tierras para trabajar. En ese tiempo, señalan, la comunidad era un pequeño rancho con pocas casas y con caminos en mal estado, sobre todo en tiempo de lluvias, y los habitantes se dedicaban a recolectar el coquito de aceite y a criar vacas, además de cultivar el campo para obtener productos para su subsistencia, como maíz y frijol, entre otros alimentos ${ }^{2}$.

2 Comunicación personal en conversaciones informales y entrevistas. 
En el transcurrir de los años, sus habitantes abandonaron paulatinamente la recolección de coco de aceite e incorporándose paulatinamente en actividades de servicios frecuentemente relacionadas con el sector turístico. La pesca no ha representado una actividad económica importante, como en otros poblados de la región, quizá porque el asentamiento se ubica a más de un kilómetro del mar. Otra de las fuentes por las que los ejidatarios han adquirido ingresos en las últimas décadas ha sido por la venta de predios, principalmente en el litoral (Gobierno del Estado de Nayarit, 2008).

Tabla 1. Evolución de la población de Higuera Blanca 1950 hasta 2010

\begin{tabular}{|cccc|}
\hline $\mathbf{A n ̃ o}$ & Hombres & Mujeres & Total \\
\hline $\mathbf{1 9 5 0}$ & 52 & 36 & 88 \\
\hline $\mathbf{1 9 6 0}$ & 101 & 90 & 191 \\
\hline $\mathbf{1 9 7 0}$ & - & - & 402 \\
\hline $\mathbf{1 9 8 0}$ & 329 & 308 & 637 \\
\hline $\mathbf{1 9 9 0}$ & 303 & 300 & 603 \\
\hline $\mathbf{1 9 9 5}$ & 292 & 272 & 564 \\
\hline $\mathbf{2 0 0 0}$ & 396 & 359 & 755 \\
\hline $\mathbf{2 0 0 5}$ & 517 & 443 & 960 \\
\hline $\mathbf{2 0 1 0}$ & 700 & 660 & 1.360 \\
\hline
\end{tabular}

Fuente: elaboración propia con base en datos de INEGI.

Engeneral,lascondiciones devidadelacomunidadhanidotransformándosepaulatinamente, logrando, por ejemplo tener un nivel de alfabetización medio. Actualmente la comunidad cuenta con escuelas de nivel básico, medio y medio superior; sin embargo, para estudios superiores las opciones más cercanas son la Universidad de Guadalajara, campus Puerto Vallarta, o la Universidad Tecnológica de Bahía de Banderas, en Nuevo Vallarta. En una encuesta realizada en 2016, para el proyecto de investigación del que forma parte este trabajo, se encontró que el $3 \%$ de los encuestados no cuenta con estudios, el $31 \%$ cuenta con estudios de primaria, $32 \%$ tiene estudios de secundaria, $18 \%$ de preparatoria, $4 \%$ cuenta con carrera técnica, $10 \%$ con estudios de grado y un $2 \%$ señaló tener estudios de posgrado. 
Los cambios más importantes en la comunidad han ocurrido a partir del año 2003 con el inició del desarrollo turístico Litibú. En primer lugar, hubo una transformación importante en cuanto a servicios y equipamiento urbano. Por ejemplo, se construyeron y ampliaron carreteras para dar un mejor acceso al desarrollo, las cuales han beneficiado también a la comunidad, y se realizaron obras de mejoramiento urbano, como empedrado de algunas calles, rehabilitación de la plaza principal y de la clínica de salud, así como equipamiento del pozo de la comunidad, obras que estuvieron contempladas en la concertación entre FONATUR y el gobierno municipal (Gobierno del Estado de Nayarit, 2008).

En segundo lugar, un acelerado crecimiento demográfico. Según los censos de población del INEGI, la comunidad ha mantenido un crecimiento demográfico sostenido desde 1950, sin embargo en los últimos 15 años han arribado a la región varias de personas provenientes de otros estados, principalmente del sur, contratadas como mano de obra para la construcción de este y otros desarrollos. Los trabajadores de la construcción empleados en el complejo de Litibú fueron alojados en Higuera Blanca. Algunos de ellos se quedaron a residir en la comunidad o en la zona en espera de más empleo. También han llegado personas por motivos diversos, que, al observar las condiciones del empleo y los salarios (considerados mejores que en sus regiones de origen), se han quedado a residir e incluso han alentado a amigos o familiares a venir a la zona. En la encuesta señalada se obtuvo que sólo el 29\% de los residentes encuestados nació en la comunidad de Higuera Blanca; el 46\% señaló ser nativo de otro lugar del estado y $25 \%$ de otro estado de la república. Así mismo, el 30\% de los encuestados señaló tener menos de diez años residiendo en la comunidad.

En tercer lugar, ha comenzado a generarse un ligero cambio en las actividades productivas. Hace más de 20 años los empleos relacionados con la actividad turística eran casi inexistentes para los habitantes de la comunidad, pues tenían que trasladarse a otros lugares con actividad turística desarrollada, como Puerto Vallarta o Sayulita. Sin embargo, a raíz de que se han consolidado los complejos turísticos más cercanos como Punta Mita y Litibú, algunos habitantes han encontrado empleo en ellos. La encuesta reportó que $13 \%$ de los informantes está empleado en servicios turísticos y que el 3\% tiene actualmente un negocio relacionado con el turismo. Por otro lado, un $63 \%$ señaló que sus ingresos están relacionados con el turismo y un $30 \%$ consideró que la totalidad de sus ingresos provienen de ese rubro. 
Figura 2. Higuera Blanca y localidades aledañas

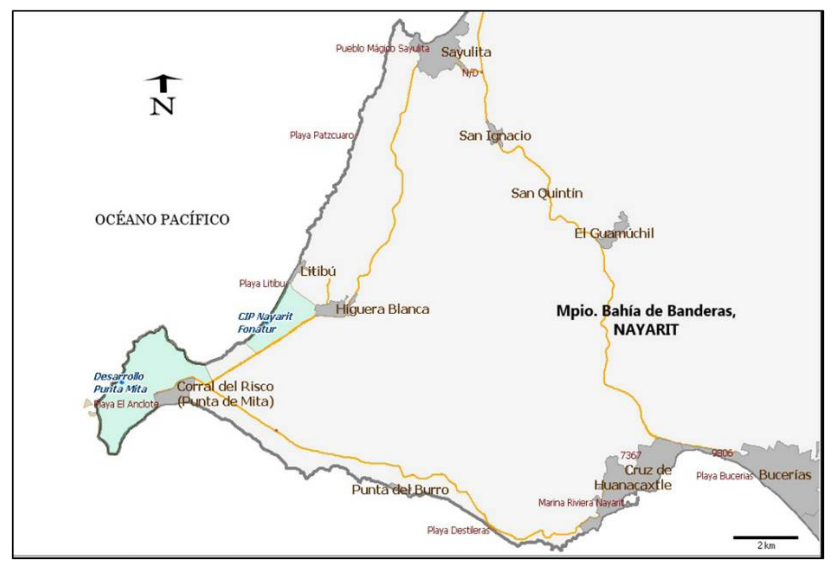

Fuente: Elaboración propia a partir del Mapa Digital de México, INEGI

Otro de los aspectos interesantes que se presenta en la comunidad de Higuera Blanca, y que relativamente forma parte de los cambios que ha experimentado en los tres aspectos señalados anteriormente, es la presencia de un grupo de extranjeros que se han asentado a un kilómetro de la comunidad, al pie de la playa, en los últimos años. Estos extranjeros son en su mayoría estadounidenses y canadienses jubilados que han tenido acceso a la compra de terrenos en esta zona y donde han construido sus casas, las cuales ocupan sólo una parte del año. Este fraccionamiento de nombre Litibú cuenta con un promedio de 20 viviendas, algunas de las cuales funcionan como hostales, y con una población fluctuante de aproximadamente 25 personas. Los habitantes de Higuera Blanca señalan que los primeros extranjeros llegaron a vivir ahí desde hace aproximadamente 25 años. Los habitantes de Higuera Blanca los denominan gringos o extranjeros, y los asocian con la imagen tradicional de los turistas; aunque hay quienes no los perciben como tales sino como residentes del lugar. Conceptualmente estas personas se ajustarían a lo que la literatura académica ha denominado turistas de segunda residencia (Hiernaux, 2005). Estos extranjeros ofrecen empleos a algunas personas de la comunidad, principalmente mujeres que hacen la limpieza de sus casas, así como también hombres que realizan mantenimiento a sus casas, muebles o automóviles. Algunas de las personas que laboran con ellos consideraron que su empleo es un empleo relacionados con el turismo. 
La información presentada hasta aquí, muestra que la dinámica social en la región, y en particular en la vida de comunidades como Higuera Blanca, se ha ido complejizando, en la medida en que un conjunto diverso de actores, individuales y colectivos, con intereses económicos, políticos y sociales han tenido incidencia en la conformación de la región. Ello ha generado ciertas condiciones de existencia particulares para cada sujeto, dependiendo de la posición que ocupa en esta estructura de posibilidades y restricciones y de una multiplicidad de prácticas, actividades y contactos sociales que definen significativamente las vivencias en torno al turismo. Es precisamente esta dimensión la que nos interesa explorar en este trabajo, la simbólica-social, es decir, los procesos de significación que constituyen el fundamento pragmático de toda práctica y acción social.

Lo que se presenta a continuación, es el análisis e interpretación de los datos generados en la indagación de campo realizada en Higuera Blanca, en abril del 2016 y en abril y mayo del $2017^{3}$. Los datos se generaron con base en una combinación de los métodos etnográfico y fenomenológico, mediante observación etnográfica y 30 entrevistas con habitantes de la comunidad, tanto originarios como avecindados, hombres y mujeres, de distintas generaciones. Las observaciones se registraron en notas de campo y las entrevistas fueron grabadas en audio y transcritas en su totalidad para su análisis. Éste se desarrolló a partir de identificar y establecer vínculos y relaciones entre diferentes tópicos que emergieron de las notas y de los testimonios de las entrevistas.

\section{Las caras del turismo y sus significaciones en Higuera Blanca}

La razón principal para realizar el estudio en esta comunidad ha sido el hecho de que, aunque no ha sido una localidad protagonista en los procesos de transformación turística de la región, actualmente experimenta un acercamiento particular a los mismos, dada la ubicación del proyecto Litibú. La intención ha sido proporcionar una interpretación de las experiencias de sus habitantes en torno a las relaciones que han establecido con el fenómeno turístico que define el destino de la región, así como indagar cómo los significados atribuidos al desarrollo de la actividad turística se vinculan a la manera en que encaran dichas relaciones. Desde una perspectiva general, puede decirse que la relación que guarda Higuera Blanca con el turismo es en realidad un conjunto de relaciones con diferentes tipos. Para sus habitantes el turismo se define a partir de, al menos, cuatro tipos que se constituyen por diversos aspectos, como los propios turistas que difieren en algunas características destacadas por ellos. Sin embargo hay otros aspectos que definen estos tipos de turismo, como su incidencia en la comunidad y en

3 El proyecto de investigación del cual forma parte este trabajo parte de un planteamiento exploratorio con enfoque metodológico mixto, que integra tanto técnicas cualitativas como cuantitativas. No obstante, este texto en particular está concentrado en la parte cualitativa. 
el entorno próximo. Se identifican así cuatro aspectos o caras del turismo que tienen diferentes relevancias en el mundo de vida de los habitantes de esta comunidad.

En primer lugar, se presenta el turismo regional tradicional y de más larga data, desde Puerto Vallarta en los 60, y su expansión paulatina por la bahía, hasta Sayulita, así como Rincón de Guayabitos o La Peñita, por ejemplo. Ello implica que la comunidad se ha familiarizado desde esos años con la actividad turística y la presencia de turistas, tanto nacionales como extranjeros. No obstante esta cara del turismo se ubica fuera de su comunidad y a cierta distancia, por lo cual puede decirse que la relación con la comunidad ha sido más bien casual. Aunque ha habido residentes que laboraron antes, o ahora, en alguno de estos destinos en actividades relacionadas con el turismo, los efectos económicos a nivel comunidad son insignificantes. Con relación a los efectos sociales, esta dimensión del turismo ha implicado un lento pero permanente encarecimiento de la vida, desde el costo del suelo hasta el de los productos y servicios. En cuanto a lo cultural, no se ha observado una influencia importante a nivel de la comunidad.

Desde la perspectiva de la comunidad, esta dimensión del turismo es vista como algo que siempre ha existido y es, en general, una fuente de empleo para mucha gente de la región, que además implica el cambio de actividades para las generaciones más jóvenes que fueron hijos de campesinos. Algo que resulta relevante para los informantes es que el turismo genere masificación y aglomeración de gente, como en Sayulita, lugar que se menciona siempre como ejemplo de lo que no quieren que ocurra en su pueblo.

Casi a la mayoría de los turistas les gusta mucho Sayulita y allá si hay muchísimos. Y allá yo creo que sí les afecta porque hay demasiados (Ama de casa, 23 años).

La idea negativa de esta masificación está asociada con la pérdida de la tranquilidad y, en ese sentido, algunosinformantesseñalaronestarorgullososdequeHigueraBlancanohayaperdido esa tranquilidad. Esta postura es una muestra de que el turismo no significa necesariamente una opción de bienestar y prosperidad para los habitantes de comunidades con potencial turístico, como la postura oficial de organismos gubernamentales y privados sugiere.

Un segundo aspecto es el turismo local o doméstico, es decir, los habitantes de poblados aledaños que durante la Semana Santa o días de asueto acuden a la playa. Algunos de estos visitantes pasan sólo algunas horas y otros llevan sus artículos para acampar. En general suelen ser de bajos ingresos y se movilizan en transporte público o en camionetas llevando sus alimentos y bebidas. A estos turistas, como los habitantes de Higuera Blanca los denominan, se les considera algo sucios y desordenados porque consumen, casi siempre, mucho alcohol. Tampoco les agrada que acostumbren acudir al poblado en traje de baño o con poca ropa. Aunque el clima de 
la región favorece el uso de prendas delgadas o poca ropa, la gente de Higuera Blanca no viste así, por ello su incomodidad al llegar a una tienda o a la parada de autobuses y encontrar a hombres y mujeres vistiendo con prendas que dejan ver mucha piel.

Vale decir que esta actividad recreativa, aunque tiene ya varios años, actualmente se ve favorecida por la cada vez menor cantidad de accesos públicos a las playas en la región, como señala uno de los habitantes "desde La Cruz [de Huanacaxtle] nada más hay una sola entrada a una playa, todas las playas que eran de uso común, ya no" (Comerciante, 52 años). De tal suerte, el vacacionista local es identificado como un actor relativamente incómodo, aunque inofensivo, y representa un tipo de "turismo" que tiene escasa relevancia para la comunidad en términos económicos y socioculturales.

En tercer lugar, se encuentra el grupo de extranjeros que viven por temporadas en el fraccionamiento anexo a la comunidad. Ellos, que también son identificados como turistas, pero de otro tipo, por parte de los habitantes, tienen una relación más directa con la comunidad. En primer lugar, porque deben cruzar por la comunidad para llegar a sus casas y, en segundo, porque tienen relaciones laborales con quienes trabajan como personal de jardinería y limpieza. Estas personas ocasionalmente colaboran con apoyos para programas de limpieza de los espacios públicos y de rehabilitación de escuelas ${ }^{4}$, lo cual es una fuente de influencia en la dinámica social de la comunidad. Se puede decir que también en el ámbito cultural han generado una incidencia menor, pues han contribuido a dar difusión al idioma inglés, principalmente con sus empleados, así como también han dado visibilidad a la tradición del Halloween desde sus casas.

Desdelaperspectivadelosinformantes destacaciertainconformidad porel hechodequeestos extranjeros no quieren tener presencia de visitantes en el espacio donde tienen sus casas y, en ocasiones,impidenelaccesoalasplayasanexasporlarazóndeque"dejanbasura"ohacenruido:

Aquí tenemos la tradición que en Semana Santa pues acudimos a acampar toda la semana allá, prácticamente es sólo en esa semana. Y pues ha habido muchas molestias de ello y hasta la policía han mandado y cosas así por el estilo, porque no quieren que vayamos. Según porque hacemos mucho relajo y cosas así. Hasta ahorita no nos han podido prohibir exactamente que vayamos, pero sí cada año es un problema porque no quieren que hagamos campamentos ahí (Propietaria de pequeño restaurante, 49 años).

Ya pusieron cámaras en toda la calle ellos. Yo estoy de acuerdo, en su casa que tengan su cámara y todo eso, porque sí se han metido canijos vagos a robarlos... En su casa yo digo que está muy bien, su cámara, pero ya pusieron no sé por dónde, por ahí... (Agricultor, 52 años).

4 Algunos de estos extranjeros, participan en la ONG denominadaAlianza de la Costa Verde, queseautodefinecomo "unareddeasociacionescivilesypersonasinteresadasquepromuevenelDesarrolloSustentableen BahíadeBanderasy CostadeNayarit”(Valverde, 2009). 
En general, la presencia de estos extranjeros jubilados genera en la comunidad una dinámica de contrastes no solo culturales sino también económicos y sociales. En este sentido, los informantes manifiestan una especie de sentimientos encontrados, dado que por un lado reconocen las buenas intenciones en las acciones de apoyo a su comunidad, pro por el otro se desconciertan ante algunos comportamientos que por consideran intrusivos y agresivos. Con relación a los anteriores, este tipo de turismo es más relevante en cuanto a incidencias dentro de la comunidad.

Tabla 2. Tipos de turismo en torno a la comunidad de Higuera Blanca

\begin{tabular}{|c|c|c|c|c|}
\hline & $\begin{array}{c}\text { Turismo } \\
\text { tradicional } \\
\text { Puerto Vallarta, } \\
\text { Sayulita y otros }\end{array}$ & $\begin{array}{l}\text { Vacacionistas } \\
\text { locales de } \\
\text { Semana Santa }\end{array}$ & $\begin{array}{c}\text { Turistas residenciales } \\
\text { Fraccionamiento Litibú }\end{array}$ & $\begin{array}{c}\text { Proyecto Litibú } \\
\text { CIP Nayarit-FONATUR }\end{array}$ \\
\hline $\begin{array}{l}\text { Interacción } \\
\text { con los } \\
\text { residentes }\end{array}$ & $\begin{array}{l}\text { - Indirecta, fuera } \\
\text { de la } \\
\text { comunidad }\end{array}$ & $\begin{array}{l}\text { Directa, } \\
\text { efímera }\end{array}$ & $\begin{array}{l}\text { Relativamente directa, } \\
\text { dentro de la } \\
\text { comunidad, por } \\
\text { motivos de convivencia } \\
\text { cotidiana }\end{array}$ & $\begin{array}{l}\text { - Indirecta, principalmente } \\
\text { fuera de la comunidad } \\
\text { Directa en la etapa de } \\
\text { construcción, aunque no } \\
\text { con el turismo en sí }\end{array}$ \\
\hline $\begin{array}{l}\text { Efectos } \\
\text { económicos }\end{array}$ & $\begin{array}{l}\text { - Ingresos por } \\
\text { empleos } \\
\text { escasos en } \\
\text { servicios } \\
\text { turísticos }\end{array}$ & $\begin{array}{l}\text { - Ingresos por } \\
\text { consumo en } \\
\text { las tiendas de } \\
\text { abarrotes }\end{array}$ & $\begin{array}{l}\text { - Ingresos por empleos } \\
\text { en mantenimiento y } \\
\text { limpieza en casas de } \\
\text { extranjeros }\end{array}$ & $\begin{array}{l}\text { - Ingresos por empleos en } \\
\text { mantenimiento y limpieza }\end{array}$ \\
\hline $\begin{array}{l}\text { Efectos } \\
\text { sociales }\end{array}$ & $\begin{array}{l}\text { Paulatino } \\
\text { encarecimiento } \\
\text { de la vida }\end{array}$ & $\begin{array}{l}\text { - Relativa } \\
\text { alteración de } \\
\text { "la } \\
\text { tranquilidad" }\end{array}$ & $\begin{array}{l}\text { - Fomento de programas } \\
\text { de limpieza en la } \\
\text { comunidad } \\
\text { - Privatización de la } \\
\text { playa } \\
\text { - Desigualdad social }\end{array}$ & $\begin{array}{ll}\text { - } & \text { Inmigración } \\
\text { - } & \text { Inseguridad } \\
\text { - } & \text { Privatización espacial } \\
\text { - } & \text { Carencia de agua potable }\end{array}$ \\
\hline $\begin{array}{l}\text { Efectos } \\
\text { culturales }\end{array}$ & $\begin{array}{l}\text { No se observa } \\
\text { influencia } \\
\text { directa }\end{array}$ & - Ninguno & $\begin{array}{l}\text { - Influencia del idioma } \\
\text { inglés } \\
\text { - Presencia del } \\
\text { Halloween } \\
\text { - Enfasis en el folklor } \\
\text { mexicano }\end{array}$ & $\begin{array}{l}\text { - Progresivo interés por la } \\
\text { lengua inglesa para el } \\
\text { empleo en servicios } \\
\text { turisticos }\end{array}$ \\
\hline
\end{tabular}

Fuente: elaboración propia con base en el trabajo de campo

El cuarto aspecto del turismo, y el más importante, que se despliega en torno de esta comunidad, es el de los enclaves turísticos, principalmente el proyecto Litibú, deFONATUR. La relación de la comunidad con éste se da básicamente a partir de los empleos que desempeñan ahí algunos habitantes; es decir, se trata inicialmente de una relación laboral. La comunidad interactúa con los turistas de este complejo sólo indirecta y externamente vía los residentes empleados en el complejo. Sus empleos son de jardinería, camarería o mantenimiento de los campos de golf. La presencia de este complejo a un costado de Higuera Blanca ha favorecido algunos matices culturales y cambios incipientes en los valores de sus habitantes, principalmente en los jóvenes, quienes han incrementado su interés por aprender el idioma inglés y por capacitarse en el sector servicios. Estos jóvenes tienden a considerar que ésta es una salida más fácil para ganarse la vida que estudiar una profesión. 
Por otro lado, aunque esos turistas suelen visitar ocasionalmente la comunidad, sólo lo hacen de forma efímera mediante paseos guiados a caballo, en cuatrimoto o en bicicleta, por lo cual no se les observa caminando e interactuando con los habitantes de la comunidad o consumiendo en los comercios de abarrotes o de alimentos, por ejemplo. Es importante subrayar también que ello se debe a que este desarrollo turístico se encuentra delimitado por un muro que impide el acceso a quienes no tienen una relación laboral o de consumo con los establecimientos del mismo. Esa disposición física y arquitectónica define parte de su relación con el entorno y, en la inmediatez, con la comunidad. Con ello la relación con la comunidad queda marcada por el sello de la segregación espacial y la exclusión más que por la interacción social y el contacto. Desde la experiencia y perspectiva de los habitantes de Higuera Blanca el muro referido despierta diferentes reacciones en ellos:

Es para privacidad, o no sé, porque pues se dice que a lo mejor quieren que uno no vea cosas. Pero qué pueden hacer que no quieren que vea (Agricultor, 52 años).

Pues yo pienso que por la privacidad propia del que está ahí, porque si tuviéramos seguridad aquí en el pueblo otra cosa sería de entrada. Ahí protegen que no haya vandalismo, entonces es como algo privado, "no pasas aquí porque a lo mejor eres mala persona"... es la seguridad pues. No puede entrar quien sea (Empleado de servicios turísticos, 39 años).

Esas reacciones, muestran la importancia que tiene esta delimitación física y simbólica para los habitantes. Estos testimonios se remiten reiteradamente a la privacidad y a la seguridad como justificantes para un aislamiento del entorno por suponerse hostil, contribuyendo así a su estigmatización. Esta es la principal característica de los enclaves turísticos (Fonseca, 2009) y que contribuye a generar segregación espacial y exclusión social. En este sentido, hay una asociación directa del proyecto Litibú con el desarrollo de Punta Mita, que es el enclave turístico de exclusividad y alto costo más representativo de la región.

Resulta interesante que, aunque la dinámica turística de Litibú no está inmersa dentro de la comunidad de Higuera Blanca, este desarrollo representa la cara del turismo que más le ha generado efectos sociales. Entre ellos destacan, además de lo mencionado arriba, la intensa inmigración generada desde su etapa de construcción, el aumento de la inseguridad relacionado con dicha inmigración, la carencia de agua potable (la cual, se rumora, se debe a que el agua se va para los hoteles y el mantenimiento de los campos de golf). Los testimonios siguientes pueden ofrecer una muestra de lo anterior:

Así como llega lo bueno también lo malo... acarrea mucha gente desconocida, que no sabes sus modales, sus intenciones. Entonces, pues sí se generan más robos, más inseguridad, más borrachillos. Antes se podía andar en las calles por las noches y no pasaba nada y ahorita ya no puedes andar tranquilo porque te sale alguien por ahí y te va a asustar o te va a robar lo poquito que traes (Empleado de servicios turísticos, 39 años). 
Se escuchan muchos rumores de que el hotel le paga al que se encarga de abrir llaves, y de mantener todo eso, para que les abra o les venda el agua a ellos (Empleado en tienda de abarrotes, 25 años).

Antes uno hacia su ramadita [en la playa], cuando era Semana Santa, y ahí pasaban los dos o tres días de la semana. Ahorita ya con el desarrollo ya no lo dejan acampar (Empleado de servicios turísticos, 39 años).

Por otra parte, a los habitantes mayores el complejo de FONATUR les trae a la mente la expropiación para el fideicomiso FIBBA de 1970. Quienes son optimistas, recuerdan que cuando se realizó la expropiación el gobierno les "regaló" casas, que aunque eran muy sencillas, pudieron hacerlas funcionales. Sin embargo, otros informantes sostienen que el gobierno sigue en deuda con ellos, pues no les ha retribuido con un pago justo como debió hacerlo en su momento. Un informante señaló que, algunas personas de la comunidad, incluido él, mantienen una demanda contra el gobierno para exigir la totalidad de la retribución, además de que no se les pagó lo justo, considerando el valor que tienen ahora las propiedades. Así, el desarrollo del FONATUR ha venido a significar una opulencia ofensiva a las puertas de su casa, pues lo que antes tenían un poco más lejos ahora está a unos metros. Ello ha provocado que las sensaciones de segregación y exclusión social se exacerben.

En síntesis, los habitantes de Higuera Blanca poseen una significación heterogénea del turismo a partir de su diversidad de experiencias. De esta manera el turismo presenta para ellos varias caras representadas por diferentes actores y dinámicas además de su relevancia en términos de efectos o incidencias en su mundo de vida. El turismo de los destinos más tradicionales, junto con los vacacionistas locales que visitan a playa de Higuera Blanca, constituyen aspectos casi irrelevantes para sus habitantes. No obstante, los tipos de turismo que se han aproximado más a la comunidad de Higuera Blanca, es decir, tanto el fraccionamiento de extranjeros norteamericanos de segunda residencia como el complejo Litibú, son los que, desde la perspectiva de la comunidad, han representado mayores incidencias en la misma. De ellas, las que más destacan son las de tipo social. En particular, los turistas extranjeros de segunda residencia aparecen como actores ambiguos que contribuyen, a partir de mayores ventajas económicas y con cierto estatus vinculado a su origen nacional, al bienestar comunitario, sin embargo también aparecen como gente con intereses de segregación y apropiación de la playa sin derecho. De igual manera, los efectos más relevantes del desarrollo Litibú, desde la perspectiva de los habitantes de la comunidad de estudio son dos y resultan paradójicos. Por un lado, representa posibilidades de mejora e ingresos, mientras por el otro evoca sensaciones de despojo y exclusión. 
Estos elementos significativamente reiterados constituyen parte de un proceso de exclusión social en el sentido de que, más allá de los elementos económicos, se pone énfasis en la multidimensionalidad y la calidad de la integración o membresía de las personas a una sociedad (Bhalla y Lapeyre, 1999). Los procesos de exclusión social en este contexto, están dibujados no sólo por la tendencia a la polarización en términos del nivel económico y la capacidad de consumo, sino además por una progresiva limitación en la toma de decisiones y el aprovechamiento de los recursos. Los residentes de Higuera Blanca, sin embargo, no viven esta situación como mercaderes racionales ni como meros espectadores, pues su participación en la lucha por la supervivencia en una estructura de progresiva y compleja polarización social viene dada en la forma en que negocian los sentidos y discursos de los modelos de desarrollo impuestos y los resignifican, pues ello es la base primordial para actuar en consecuencia.

\section{Conclusiones}

El análisis significativo de las prácticas y comportamientos específicos nos deja comprender que las personas no actúan apegadas a los guiones de los grandes proyectos concebidos desde una lógica racional simplificadora. El hecho que exista una gran estructura de limitaciones sociales y materiales, no determina que los actores tengan una sola opción. Los residentes de las comunidades que han sido sitiados por proyectos turísticos, deberían poder decidir sí el turismo es o no es la opción que más les conviene. Los actores del caso de estudio, no han tenido la oportunidad de decidir si desean o no, que su territorio se convierta en un espacio que albergue enclaves turísticos. Sin embargo, ellos han mostrado la capacidad para objetar el discurso oficial en el que se asume que el deseo natural es vivir de los beneficios de un destino turístico, pues esos beneficios resultan ridículos al lado de las ventajas de que disponen otros actores y frente a los propios perjuicios.

De alguna manera, estas personas se hallan envueltas en una dinámica con una fuerza determinante, quizá imparable, y es la que está definiendo de forma dramática sus condiciones de integración o exclusión social. Los recursos con los que cuentan son, por un lado, su capacidad de organización mediante la cual pueden decidir las formas de negociar el ingreso o rechazo y, por el otro, hacerse escuchar para ser tomados en cuenta en la gestión de estos proyectos en todos sus aspectos.

Uno de los aportes de este trabajo, es precisamente mostrar cómo desde una aproximación metodológica alternativa, cualitativa, se tiene acceso a una mayor comprensión de las realidades sociales en estos contextos. Ello consintió no sólo en identificar algunos efectos socioculturales del fenómeno turístico, sino también entender el componente de los elementos del proceso desde la perspectiva de sus protagonistas. Por otro lado, nos permitió reflexionar que no solo las percepciones de 
las sociedades receptoras de turismo son heterogéneas, en el sentido que lo postularon Ap y Crompton (1993), sino que tampoco los turistas son un grupo monolítico y, por tanto, las representaciones que se tienen de ellos varían de manera importante.

Es decir, no se presenta igual, y por lo tanto no significa lo mismo, el turista mochilero, que el turista residencial o el turista de exclusividad. En otras palabras, hay de turistas a turistas: con el turista que acude al proyecto Litibú los residentes de Higuera Blanca tienen poco o nulo contacto, por lo que es difícil hacerse alguna representación de él. El turista que asiste a Sayulita, es el turista 'hippioso' que se adapta a la comunidad, que no requiere de servicios especializados, por ello lo observan con mayor frecuencia, pues al mismo tiempo es sitio frecuentado por los pobladores de Higuera Blanca como espacio de ocio. El snowbird es un vecino distante, pero vecino al fin y al cabo. El turismo doméstico es incómodo pero sólo lo ven pocas veces al año.

En términos generales, estas estampas de la vida cotidiana, experiencias, emociones, predisposiciones, etc., resultan útiles para repensar desde una mirada más profunda y cimentada los beneficios y afectaciones de este tipo de proyectos. Si bien a los actores que nos dieron sus testimonios les ha ido relativamente "bien", por considerar que su suerte ha mejorado, es importante llamar la atención de que estos desarrollos deben tomar en cuenta un beneficio justo a los pobladores. El enorme despliegue de capitalización en esa región, como en muchas más, no ha generado beneficios realmente equitativos. El desarrollo turístico en México debe considerar las necesidades de las comunidades a través del diálogo y la negociación y no darlas por supuestas. Los proyectos de desarrollo turístico diseñados desde un modelo tradicional tienden a generar más efectos negativos cuando se pierden de vista los aspectos humanos y la riqueza en las formas de entender y definir el mundo.

\section{Bibliografía}

Arias-Hidalgo, D., \& Méndez-Estrada, V. H. (2015). Differences in housing, lodges, language, kinship and perception of tourism in Bribri communities with varying levels of geographic isolation (Talamanca, Costa Rica). Cuadernos de Investigación UNED, 7(2), 119-129.

Ayuntamiento de Bahía de Banderas. (2014). Plan de Desarrollo Municipal Bahía de Banderas 2014-2017. Recuperado de http://www.oromapas.gob.mx/transparencia/ numeral6/PLAN_DE_DESARROLLO_2014-2017.pdf

Baños Francia, J. A. (2014) Consideraciones sobre la gestión metropolitana en México. Acercamiento al caso de la Bahía de Banderas, Trace, 64, consultado el 29 junio 2017. URL: http://trace.revues.org/1256 
Bassi, D. Á., \& Cardona, J. R. (2015). Efecto de los impactos económicos, culturales y en las actitudes de los residentes de Punta del Este. European Journal of Applied Business and Management, 1(2).

Berger, Peter. L. y Thomas Luckman. (1968). La construcción social de la realidad. Buenos Aires: Amorrortu, 1988.

Berrezueta, L. A. O., Grunauer, M. S. N., Carrillo, J. A. R., Contreras, O. S. O., \& García, M. C. (2017). Commune perceptions of the sociocultural impacts derived from rural tourism: Bellavista case, Ecuador. RIAT: Revista Interamericana de Medioambiente y Turismo, 13(1), 15-27.

Bhalla, A. S. y Frédéric L. (1999). Poverty and Exclusion in a Global World. New York: St. Martin's Press.

Blumer, H. (1969). Interaccionismo simbólico. Perspectiva y método. Barcelona: Hora, 1982.

Brunt, P., y Courtney, P. (1999). Host perceptions of sociocultural impacts. Annals of Tourism Research, 26: 493-515.

Cardona, J. R., \& Serra Cantallops, A. (2014). Inicios del turismo y actitudes de los residentes: El caso de Ibiza-España. Estudios y perspectivas en turismo, 23(1), 01-22. Castro, U., Fonseca, M. A. y López, R. (2015). Desarrollo y turismo: la preeminencia del modelo de enclave en México y sus efectos en el desarrollo local, en Arnaiz Burne, S. y Judith Juárez (coord.) Desarrollo, Crisis y TURISMO. Puerto Vallarta: U de G., pp. 28-45.

Chen, S., and C. Raab. (2012). "Predicting Resident Intentions to Support Community Tourism: Toward and Integration of Two Theories." Journal of Hospitality Marketing \& Management, 21 (3): 270-94.

Choi, H: C. y Murray, I. (2010). Resident attitudes toward sustainable community tourism, Journal of Sustainable Tourism, 18 (4): 575-594

Departamento Agrario. (19 de enero de 1953). Resolución sobre dotación de ejido al poblado de Higuera Blanca, en Compostela, Nay. Diario Oficial, p. 13-14.

Departamento Agrario. (6 de agosto de 1940) Resolución en el expediente de dotación de tierras al poblado de Sayulita, Estado de Nayarit. Diario Oficial, 2a sección, p. 15-16. Fernández-Agraz, C. (2014). El turismo como factor de transformación del sector agrícola de Bahía de Banderas, Nayarit. Tesis de doctorado no publicada, Universidad de Guadalajara.

Fishbein, M., \& Ajzen, I. (1975). Belief, Attitude, Intention, and Behavior: An Introduction to Theory and Research. Reading, MA: Addison-Wesley FONATUR. (2006) Libro blanco. Centro Integralmente Planeado Nayarit (CIP Nayarit). Disponible en: www.fonatur.gob.mx. Acceso el 17/02/2016.

Fonseca, M. (2009) Punta Mita en la dinámica del desarrollo turístico regional. El periplo sustentable $16,(85-108)$.

Garfinkel, Harold. (1967). Studies in Ethnomethodology. New Jersey: Pretince Hall. 
Geertz, Clifford. (1973). La interpretación de las culturas. Barcelona: Gedisa, 2003. Gobierno del Estado de Nayarit (13 de diciembre de 1989) Decreto 7261. Se crea un nuevo municipio en la zona sur del territorio del actual Compostela, que se denominará Bahía de Banderas, quedando integrado en la base de la administración política y administrativa del municipio libre en el estado de Nayarit. Periódico Oficial, Segunda sección, Tomo CXLVI, No. 48.

Gobierno del Estado de Nayarit (26 de enero de 2008) Plan parcial de urbanización CIP Polígono Litibú Bahia De Banderas, Nayarit. Periódico Oficial, Sección decimosegunda, Tomo CLXXXII, No. 13.

Gómez Encarnación, E. (2014). El Guamuchil y sus fiestas patronales. Disponible en: https://www.bahiadebanderas.gob.mx/?page_id=2763. Acceso el 17/04/2017. Gómez Encarnación, E. (2015). Sayulita, antes Santa Cruz Saloc. En Tribuna de la Bahía, 24 de febrero de 2015. Dsiponible en: http://badebatribuna.blogspot.mx/2015/02/ en-los-tiempos-desayulita-antes-santa.html. Acceso el 10/04/2017.

Gómez, A. (2010). Nayarit como un estado de múltiples dimensiones migratorias. Revista Fuente, 1: 15-21.

González Herrera, M. R. (2011) "Impactos percibidos del modelo turístico urbano en Remedios y Caibarién, Cuba. Retos para la sostenibilidad" Revista de Análisis Turístico, 11, 23-34.

Hiernaux, D. (2005). La promoción inmobiliaria y el turismo residencial: el caso mexicano. Scripta Nova. Revista electrónica de geografía y ciencias sociales, IX, núm. 194 (05). Disponible en: http://www.ub.es/geocrit/sn/sn-194-05.htm. Acceso el 4/09/2015.

Hunt, C., \& Stronza, A. (2014). Stage-based tourism models and resident attitudes towards tourism in an emerging destination in the developing world. Journal of Sustainable Tourism, 22 (2): 279-298.

Hunter, W. C. (2013). "Understanding Resident Subjectivities towards Tourism Using Q Method: Orchid Island, Taiwan.” Journal of Sustainable Tourism, 21 (2): 331-54. Jiménez, M. A. A., Méndez, M. F. P., \& Rodríguez, D. A. F. (2017). Impactos socioculturales ambientales del desarrollo turístico en Mezcales, comunidad del municipio de Bahía de Banderas, Nayarit. Revista Multidisciplinaria de Avances de Investigación, 3(1), 1-10.

Kim K., Uysal M., Sirgy M. J. (2013). "How Does Tourism in a Community Impact the Quality of Life of Community Residents?” Tourism Management, 36:527-40.

Lee, T. H. (2013). Influence Analysis of Community Resident Support for Sustainable Tourism Development. Tourism Management, 34:37-46.

Marins, S. R., Mayer, V. F., \& Fratucci, A. C. (2015). Impactos percibidos del turismo. Estudios y Perspectivas en Turismo, 24, 115-134. 
Márquez, A; Ocampo, J. y Ramos, M. (2010) Los municipios sureños de la Riviera Nayarit, México: un breve análisis sobre su dinámica demográfica. En Meza Ramos, E. y Lourdes C. Pacheco (Coords.) De aquí, de allá. Migración y desarrollo local, Tepic: UAN, pp. 157-182.

Mathieson, A. y Wall, G. (1982). Tourism: Economic, physical, and social impacts, Longman, London and New York.

Mendoza Ontiveros, M. M., \& González Sosa, J. C. (2014). Impactos socioculturales del turismo en el Centro Integralmente Planeado Loreto, Baja California Sur, México. Percepción de los residentes locales. Teoría y Praxis, (16).

Milito, M. C., Juniro, S. M., \& Alexandre, M. L. (2015). Factores que influyen en el apoyo que los residentes dan a los megaeventos. Estudios y Perspectivas en Turismo, 24, 153-171.

Monterrubio, J. C., Sosa, A. P., \& Josiam, B. M. (2014). Spring break e impacto social en Cancún, México. Un estudio para la gestión del turismo. Turismo y Sociedad, 15, 149-166.

Moro Ingeniería S.C. (2002) Manifestación del impacto ambiental en su modalidad regional para el Proyecto Litibú, Nayarit. México: SEMARNAT.

Nunkoo, R., S. Smith, and H. Ramkissoon. (2013). Residents' Attitudes to Tourism: A Longitudinal Study of 140 Articles from 1984 to 2010. Journal of Sustainable Tourism, 21 (1): 5-25.

Parra-Camacho, D., \& Bastías, D. D. (2013). Percepción de los residentes sobre el impacto socioeconómico de un evento deportivo: análisis de segmentos y perfil del residente. Journal of Sports Economics \& Management, 3(1), 4-3

Plan Municipal de Desarrollo de Bahia de Banderas. (2011-2014). Disponible en: http:// www.oromapas.gob.mx/transparencia/numeral6/pmd_2011_2014.pdf. Acceso el 16 de abril de 2016.

Ramón Cardona, J., \& Álvarez Bassi, D. (2016). Percepción de los impactos socioculturales en Punta del Este (Uruguay). El turismo y la experiencia del cliente: IX Jornadas de Investigación en Turismo (2016), 515-536

Rodrigues Marins, Simony, Feder Mayer, Verônica, \& Fratucci, Aguinaldo Cesar. (2015). Impactos percibidos del turismo: Un estudio comparativo con residentes y trabajadores del sector en Rio de Janeiro -Brasil. Estudios y perspectivas en turismo, 24(1), 115-134. Recuperado en 14 de julio de 2017, de http://www.scielo.org.ar/scielo. php?script=sci_arttext\&pid=S1851-17322015000100007\&lng=es\&tlng=es Salazar-Peralta, A. M. (2013). Dinámicas psicopolíticas el turismo internacional y las segundas residencias en la Riviera Nayarit. En C. O. Bazán, Enfoques antropológicos sobre el turismo contemporáneo. México: UNAM-Instituto de Investigaciones Antropológicas, pp. 111-129. 
Sánchez-Fernández, M. D., \& Cardona, J. R. (2016). Impacto de las actitudes de los residentes sobre el grado de aceptación del turismo de playa, el turismo de deportes, el turismo de naturaleza y el turismo cultural. Podium sport, Leisure and Tourism Review 5(2), 56-75

Schütz, Alfred. (1932). La construcción significativa del mundo social. Introducción a la sociología comprensiva. Barcelona: Paidós, 1993.

Secretaría de Economía de Nayarit (1952). Séptimo Censo General de Población 1950. Dirección General de Estadística.

Secretaría del Patrimonio Nacional (13 de noviembre de 1970). Decreto que declara de utilidad pública el desarrollo habitacional y turístico en los terrenos que circundan la Bahía de Banderas, ubicados en las costas de los Estados de Nayarit y Jalisco y el mejoramiento de varios centros de población, para lo cual se expropian en favor del Gobierno Federal, varias superficies ejidales del Municipio de Compostela, Nay. etc. Diario Oficial de la Federación, Tomo CCIII, No. 15, pp. 4-5.

Sedesol (2010a) Microrregiones. Sistema de Apoyo para la Planeación del PDZP. Disponible en: http://www.microrregiones.gob.mx/catloc/contenido. aspx?refnac $=180200041$. Acceso el 11/02/16 Sedesol (2010b) Microrregiones. Sistema de Apoyo para la Planeación del PDZP. Disponible en: http://www.microrregiones.gob.mx/catloc/contenido. aspx?refnac $=180200118$. Acceso el 17/04/16

Sharpley, R. (2014).Host perceptions of tourism: a Review of the research, Tourism Management. 42: 37-49

Valverde, M. (2009). En defensa del paisaje construido. El caso de Bahía de Banderas, Nayarit. Topofilia. Revista de Arquitectura, Urbanismo y Ciencias Sociales, I (3): 1-18. Vargas, A. Porras, N. y Plaza, M. A. (2011). Residents' Attitude to Tourism and Seasonality. Journal of Travel Research. 53, (5), $581-596$

Weber, M. (1922a). Economía y sociedad, Fondo de Cultura Económica, México, 1997. Weber, M. (1922b). Clase, estamento y partido, en Claudio Stern (comp.) La desigualdad social II. Teorías de la estratificación y la movilidad sociales. México: SEPSENETAS, 1974, pp. 7-29.

Woosnam K. M. (2012). Using Emotional Solidarity to Explain Residents' Attitudes about Tourism and Tourism Development. Journal of Travel Research, 51 (3): 315-27 Yu C. P., Chancellor H. C., Cole S. T. (2011). Measuring Residents' Attitudes toward Sustainable Tourism: A Reexamination of the Sustainable Tourism Attitude Scale. Journal of Travel Research, 50 (1): 57-63

Zamani-Farahani, H., \& Musa, G. (2012). The relationship between Islamic religiosity and residents' perceptions of sociocultural impacts of tourism in Iran: Case studies of Sare'in and Masooleh. Tourism Management, 33(4): 802-814. 